\title{
INSTITUTION ET FORMATION
}

L'insitution est le cadre, un cadre qui s'adosse toujours à un dejàla, et il faut encore y faire autorite. Une formation d'enseignants, comme toute formation, requiert que place soit faite à la mémoire: reconstruction, récit qui met en forme et contribue à former. Place aussi à l'écart, au dialogue, à la décision réfléchie. L'inscription de la philosophie permet ces perspectives. L'institution de formation a aussi partie liee avec l'école publique: a-t-on pris la mesure de l'exigence étrangère à l'autonomie universitaire, de la läcité, a-t-on mesuré la difficulté d'assumer la question de la culture comme références communes et expérience singulière?

Sous ce titre je voudrais poser deux questions, une "question d'école" et une question d'actualité. L'actualité est marquée par la mise en place d'un nouveau dispositif de formation des maitres. Nous devrions assister ou participer à la mise en oeuvre d'une idée, aussi vieille que le plan Langevin-Wallon qui, comme on le sait, résultait de l'écroulement des institutions de la IIIe République, des épreuves de la guerre et de $\mathbf{l}^{\prime}$ occupation, de la suppression des écoles normales d'instituteurs par l'Etat français installé a Vichy et des espoirs que suscitait la victoire remportée sur la barbarie nazie. Dans une France libérée, on a projeté pour la première fois de concevoir un "corps unique" de maîtres qui seraient tous formés à l'université. Est-ce bien ce projet qui se réaliserait aujourd'hui dans un pays qui, après l'explosion scolaire des années 60 et la découverte des limites de la démocratisation, s'apprête à affronter les échéances de la construction européenne en se proposant de conduire plus de trois quarts de ses enfants au niveau baccalaureat? La formation est plus que jamais à l'ordre du jour et le bouleversement des institutions calmement envisagé selon une démarche pragmatique qui ne suscite guère de débats et, semble-t-il, assez peu d'enthousiasme.

D'où la question d'école. Institution, formation: quel lien entre les deux notions? Quelle institution est requise par telle ou telle idée directrice de la formation? Quelle conception de la formation découle de tel ou tel type d'institution? Nous le savons d'expérience, n'importe quelle formation n'est pas possible dans n'importe quelle institution. On est tenté de relever, en premier lieu, à quel point l'institution limite le champ du possible ou influe sur les effets du projet de formation. Pour 
prendre l'exemple des institutions les mieux installées dans le paysage qui est en train de se défaire sous nos yeux, les écoles normales d'instituteurs, le statut de fonctionnaires-stagiaires de leurs "étudiants" pèse considérablement sur le projet des "formateurs" que sont les professeurs d'écoles normales, fonctionnaires-titulaires eux, mais sans statut spécifique reconnu! Une seule expérience suffit à rendre perceptible le poids et le jeu de l'institué dans cette affaire: le franc succès que connaissent les "intervenants extérieurs" qui viennent moins briser la monotonie d'un enseignement ordinaire que rompre le jeu à somme nulle des places distribuées par la donne règlementaire dont, dans le rapport pédagogique quotidien, chacun fait mine d'être affranchi. D'où une première conséquence: l'ordinaire de l'institution pèse sur les dynamiques, les enthousiasmes, les formes de mobilisation et les espoirs contenus dans le projet de (se) former. On peut y voir l'une des sources de l'idée "moderne" qui vise à transformer les fonctionnaires en missionnaires et à remplacer la logique de l'institution par celle de la mission. Au temps indéterminé d'une formation interminable et "interminee" se substitue le temps court du stage, intensif, mobilisateur qui peut aussi bien se dérouler dans des installations précaires, campements plantés au plus près du "terrain", plutôt qu'en ces séminaires à l'architecture imposante et centenaire construits dans les préfectures. On aura reconnu là une rhétorique qui a couru depuis les années 1970 où s'est mis en place la formation continue des instituteurs, marquée par l'exigence de la rénovation pédagogique, jusqu'à la création des MAFPEN, au début des années 80 fondée sur la critique des institutions de formation contenue dans le rapport De Peretti. L'urgence de l'action, la localisation au plus près du terrain, la recherche d'une dynamique nouvelle, tout concourt à la promotion de l'instituant contre l'institué, à une apologie de la formation confondue avec l'innovation contre une image de l'institution qui a tous les caractères d'un "establishment."

\section{L'instituteur et la norme}

Mais, en réalité, la formation n'est pas une action, une liberté, une initiative qui viendrait s'inscrire dans un cadre, un contexte, une administration, un appareil qui en limiterait ou en pervertirait les effets. Le jeu est bien plus complexe entre l'une et l'autre.

D'une part, l'institution, dans son actualité, résulte d'une histoire qui, peu à peu, lui a donnésa "forme". Elle-même produit d'un processus, l'institution est le déjà-là de la formation. Elle a le mérite de répondre en acte à la question vertigineuse: qui formera les formateurs? Dans 
cette optique, l'institution est, au principe de toute "réforme", comme le fond sans lequel elle ne saurait faire émerger le dessin de sa forme, le dessein de son projet. De plus, l'institution est dispensatrice de moyens, de locaux, de matériels, d'outils et de techniques qui leur sont associés, elle joue comme un réservoir de procédures disponibles pour le "formateur". En clair, s'il se distingque par sa capacité à s'investir, comme on dit, c'est, au moins, à partir d'un investissement préalable.

Enfin l'institution est l'horizon même de la formation dans la mesure où cette dernière a pour tâche d'incorporer les novices, en cours de formation, dans le corps des fonctionnaires de l'institution. Ces relations, que je schématise, entre l'institution et la formation sont contenues dans le terme d'instituteur auquel on entreprend aujourd'hui de substituer celui de professeur d'école. S'y conjuguait le sens vieilli d'institution (ce qui institue les enfants, c'est-à-dire les éduque en les instruisant) et l'idée que l'école est une institution publique sinon une affaire d'Etat. La tâche première de l'institution école était donc de former ses instituteurs. C'est pourquoi les Conventionnels ont décrété la création de l'Ecole normale, en l'An III de la République. C'est pourquoi, à peu près un siècle plus tard, la IIIe République a voté des lois pour que soient crées des écoles normales d'instituteurs dans tous les départements. Ces mêmes institutions dont aujourd'hui, cent ans après, on décide l'absorption dans l'université.

\section{L'institution, lieu de mémoire}

Il faut donc s'interroger de nouveau sur les conditions de possibilité de la formation des enseignants par et pour une institution publique. C'est-à-dire chercher ce qui permet de distinguer une formation normale mais non normalisatrice, la formation d'un corps enseignant qui ne soit pas une congrégation, une formation légitime où l'état n'outrepasse pas ses droits et ses prérogatives. A mon sens, former n'est pas normer, reproduire à l'identique, agréger à une congrégation, si et seulement si l'institution de formation est un lieu de mémoire. Un lieu qui fait place à l'urgence d'une réflexion sur la mémoire dans la formation. C'est-à-dire à l'histoire, à la connaissance des institutions, à la philosophie (entendue comme le travail produit à l'articulation de l'initiation à une culture et de l'effort pour penser soi-même). La seule formation légitime dans une institution publique -ce qu'est l'école et, au premier chef l'école obligatoire- est celle qui explicite et règle non seulement son rapport aux lieux (l'ensemble des institutions politiques et des instances sociales) mais aussi son rapport au temps. A la différence 
de ce qui se passe en d'autres lieux (familles, églises, partis, etc...) la formation scolaire (qu'elle se présente sous forme de programmes, d'objectifs, de projets, ne change rien à l'affaire) ne consiste aps en romans d'apprentissage, en histoires que l'on raconte aux néophytes parce qu'elles recèlent une "vérite", en exercices que pratique le novice pour parvenir à la révélation.

Le terme de mémoire désigne l'effort nécessaire, dans une institution de formation laïque et publique, pour comprendre l'ambivalence du temps dans la formation. La formation, en effet, échappe à sa mise en forme institutionnelle parce qu'elle est toujours, à des degrés divers, un parcours erratique, un voyage aventureux. Les discours privés ou sociaux sur la formation viennent, dans l'après-coup, inscrire ces détours dans un ordre et le contresigner à la manière des récits autobiographiques et des romans de formation. Comme les voyages ne forment la jeunesse que si elle ne s'est pas perdue en chemin, la formation n'institue que dans l'après-coup d'un récit qui mène bien quelque part (ou, si l'on veut et plus cavalièrement, la formation mène à tout à condition d'en sortir). L'écriture, l'ordre du récit, la signature ont ici partie liée avec une logique stratégique (celle des programmations, des cursus, des objectifs): ils dessinent un possible ou un accompli. Quant à la formation reelle, elle est grosse de tactiques, de feintes, de ruses, de violences et de réconciliations qui s'évanouissent dans l'irréversible flux du temps: le cursus comme le récit rationalisent le cours des choses. Ce qu'on appelle formation, notamment dans le langage des "formateurs" et des politiques, c'est ce qui permettrait de faire l'économie du désordonné, du tactique, du conflictuel au bénéfice d'un récit écrit à l'avance pour l'autre, mémoire ré-ordonnée des récits de tous ceux qui l'ont déjà précédé dans l'institution.

Une institution est donc un lieu de mémoire lorsqu'à ce pouvoir de fait qu'elle tient de décider des cursus et de définir des rôles, les places et les parcours de ceux qu'elle entend former, elle ajoute ce souci de l'autre, de ses écarts, bref ce souci de faire autorité, c'est-à-dire de faire place à l'incertitude en refusant de décider à la place de l'autre. Faire autorité, c'est reconnaître les limites de l'autorité déléguée par l'institution, prendre le risque d'inventer une manière d'occuper la place et, en conséquence, d'accepter que l'autre vous réponde comme il l'entend (et non pas comme vous l'y autorisez à l'avance). Cette manière de faire a partie liée avec l'écriture: elle invente, s'inscrit dans une place "blanche" stylise et s'ouvre ay risque des lectures plurielles ou divergentes. La position traditionnelle de l'enseignant est moins celle de l'auctor que du lector. commentateur légitime, gardien des lectures orthodixes, 
donneur de leçons. Dans cette question de places le problème de l'autorité toujours s'obscurcit: est-elle seulement déléguée par l'administration (et un pion y suffirait aussi bien)? est-elle le fait de quelqu'un qui ne s'autorise que de lui-même (mais pourquoi diable alors est-il fonctionnaire)? Cette incertitude explique sans doute le succès de la métaphore du contrat dans le champ pédagogique sur lequel je reviendrai dans un instant. Reste que le rapprochement entre l'autorité et le "style" pose doublement problème. Cette singularité inscrite dans le poste de travail fait désordre dans le bel agencement des organigrammes. Et ses succès sont bien aléatoires, variables au fil du temps: on n'a pas la grâce tous les jours ouvrables de l'année scolaire. Contre ce double risque du désordre et du manque d'inspiration, l'institution joue comme une garantie justement parce qu'elle matérialise dans son architecture la mémoire des coups accomplis, des aventures passées et des tempêtes traversées, des procédures "qui marchent", des limites qu'il vaut mieux ne pas chercher à dépasser. Seul celui qui habite l'institution peut s'autoriser à jouer avec les habitus professionnels, à prendre le risque de ce qui ne se fait pas. Telle est la vertu de l'institution, permettre de faire, d'introduire la création dans l'exercice, le poème dans la leçon.

\section{La culture ou le contrat}

Ces remarques tracent, me semble-t-il, des lignes de dissensus à l'intérieur de l'institution même si ceux qui y traveillent ne tiennent pas nécessairement à les expliciter quotidiennement.

1. Un certain discours sur l'instruction comporte souvent une opération de dénégation du travail sur la mêmoire des sujets institués, tâches qui revient en premier lieu à l'institution. Ce dressage de l'animal humain destiné à lui faire une "mémoire" en le rendant apte à tenir des promesses dont parlait Nietzsche, cette inculcation des habitus qu'analysent les sociologues est bien son affaire propre. C'est elle qui se chargent, dans son fonctionnement quotidien, de graver dans les individus ces traces mnésiques qui constituent la mémoire morte par laquelle elle se perpétue. Les propos "cultivés" sur l'instruction sont, au sens propre, amnésiques et se déploient dans l'oubli des conditions de l'apprentissage de la culture: "Nous avons toujours su lire: nous ne pouvons pas nous rappeler le temps où nous ne savions pas lire... Notre enfance nous semble faite d'après-midi lents, de soirs tombants dans une salle de classe, de lampes coniques suspensdues au dessus de nos têtes, dans la jaune lumiere desquelles, toujours, nous lisions..." écrit justement Danièle Sallenave. En ce sens, il est vrai que la culture, c'est ce qui reste quand 
on a tout oublié. Et la pédagogie c'est le contraire.

2. D'autres vont s'imaginant que les institutions, comme les sociétés, se pourraient gouverner par décret. Or l'institution, arbitraire ou non, légitime ou non, est déjà là, contrainte d'abord avant que d'être, éventuellement, obligation. Dire que l'institution ne relève pas d'abord d'un contrat librement consenti c'est rappeler qu'il y a, au principe du rapport pédagogique, un rapport de forces. La rhétorique du contrat s'aveugle à vouloir le dénier et inventer toutes sortes de dispositifs sophistiqués pour fabriquer de l'adhésion. Or le travail pédagogique consiste justement à faire autorité c'est-à-dire à ouvrir les voies non d'une soumission contrainte mais de l'invention d'une manière propre d'occuper sa place en un lieu institué. Ce sont, à proprement parler les chemins de l'émancipation. L'écolier, l'éleve, l'étudiant n'est pas un individu libre de contracter. Dès lors qu'est reconnu le droit de l'Etat à instruire, la structure règlementaire de l'institution fait du contrat une fiction ou pire une machination. Il faut être singulièrement aveugle pour présupposer que l'exercice de l'autorité enseignante est contradictoire avec la possiblité pour l'élève selon ses propres voies et d'inventer le style de son émancipation dans le partage de la culture que l'institution a pour charge de transmettre.

3. Au coeur de ce dissensus, en effet, opère le conflit des interprétations sur les relations entre l'école et la culture, sur le rôle de l'école dans le rapport des générations. Une lecture socio-historique insiste sur l'ensemble des déterminations qui caractérisent le style de la formation dispensée à un moment donné, la configuration des relations entre l'école, la culture et la société propre à une formation sociale donnée. La lecture philosophique s'oppose selon les deux version qu'on vient de rappeler sommairement. Aux historiens et aux sociologues, les uns objectent que l'accès au savoir et à la culture ne requiert rien d'autre, qu'un maître qui transmet son savoir à des disciples. Que ce maître soit, par exemple, fonctionnaire de l'Etat est pour cette philosophie quasi indifferent. Les autres sont tout aussi peu soucieux des conditions historiques et sociales de leur activité d'enseignants, persuadés qu'ils sont que le premier acte de leur mission consiste à fonder, à insistuer la relation pédagogique comme s'ils transmettaient la culture dans une sorte d'état de nature. Comme si les dimensions hsitoriques et politiques du savoir et de sa transmission étaient, le jour de la rentrée des classes, mises entre parenthèses, à la merci du pouvoir de contracter des sujets. Ils se repésentent les savoirs, les relations et l'institution sous des formes juridiques. On connaît le prix de cette philosophie: les conflits incessants que suscite un rapport de forces déniées obligent à ajouter sans cesse des 
avenants au pseudo-contrat fondateur et on passe plus de temps à "instituer" (a réglementer à l'infini) qu'à instruire ou enseigner. Cette représentation angélique et apolitique secrète une prolifération de droits (droits de l'enfant, droits de l'éleve) qui jamais n'en finissent avec une histoire oubliée: celle de l'éducation comme entreprise d'intégration de l'enfance dans un monde et une culture dont les adultes sont responsables (et non simplement contractants ou partenaires). Reste que ce conflit des interprétations est irréductible que suscite à la fois le poids de l'institution et la liberté des acteurs. Ce dissensus est bien une chance et une tâche à la fois: il rend possible une sorte d'impératif herméneutique qui oblige le formateur à une éthique de la parole enseignante, seule garantie que, dans une institution qui relève de la sphère publique, la formation est bien autre chose qu'une profession de foi, une normalisation, une inculcation, un embrigadement.

\section{La mémoire au present}

Si la méfiance à l'égard de l'institution tient à sa tendance à l'inertie et à l'auto-reproduction, au corporatisme qu'elle sécréterait, les charmes de l'idée modernes de formation sont liés à des images d'ouverture, de modernité, de dynamisme. Bien entendu, pour être sensible à de tel charmes il faut un patient travail d'oubli et d'aveuglement: il n'est pas nécessaire d'être un sujet supposé savoir pour déceler les innombrables retours du refoulé de l'institué, du religieux laïcisé, dans les enthousiasmes que provoque parfois la formation. Combien de chemins de Damas pédagogiques, de conversion, de révélations avec leur cortège de sectes, de prophètes et de gourous). Je tiens cette opposition entre institution et formation pour une erreur de perspective et je me demande plutôt, à l'aube de la création de nouvelles institutions "universitaires" de formation quelles significations nouvelles vont prendre les rapprots entre pédagogie et philosophie.

1. L'université, on le sait depuis longtemps, est apte à former des professeurs, des maîtres qui professent ceci ou cela, selon ce que leurs maîtres leur ont enseigné. Il s'enseigne bien des choses et de bien des manières dans des Universités légitimement autonomes, et il s'y soutient librement toutes sortes de thèses. Mais savoir ce qu'il faut transmettre, c'est aussi décider de ce qu'il ne faut pas enseigner. L'enseignement élémentaire, l'instruction des enfants, l'initiation des jeunes générations supposent le secret, le délai, le report à plus tard de certains savoirs. On ne remarque peut-être pas assez, à cet égard, que l'Université n'a pas de programme. On ne saurait le lui reprocher: c'est la 
conséquence de sa vocation à l'Universel. On peut tout y enseigner et, dans ce lieu où l'apprenti est auprès du maître, au contact de la recherche en train de se faire, on peut apprendre en cherchant. De ce point de vue, l'Université est plus proche de la maternelle que de l'école élémentaire ou du college. Elle n'est d'ailleurs pas plus obligatoire qu'elle. Mais il y a plus et en cela l'Université se dintingue radicalement du reste du "systeme scolaire": elle est autonome et ses enseignants constituent une corporation dont les membres s'élisent entre eux.

Dès lors que l'on choisit, pour les instituteurs de l'école obligatoire comme pour les professeurs, une formation à l'Université on cesse certes de se replier frileusement sur une conception normalisatrice et unificatrice de l'entrée dans le métier d'enseignant. Mais, si l'on veut rester conséquent avec les principes de l'Ecole républicaine, il faut être alors résolument vigilant sur les procédures de régulation du pluralisme des contenus professés, les modes de relation entre maîtres et disciples, les règles du jeu et les instances d'arbitrage.

2. Une seconde interrogation concerne le rôle que jouent les institutions comme lieux de mémoire. Quelle place l'Institut universitaire fera-t-il à la mémoire pédagogique? Que l'on entende bien, un lieu de mémoire est nécessairement un lieu d'oubli. La mémoire vive ne relève pas seulement de l'archive, du conservatoire ou de la bibliothèque. Mémoire signifie mise en perspective. Une telle opération suppose un travail de problématisation, d'où le problème de la capacité de la philosophie à faire là acte de présence.

"Acte de présence" serait-ce trop peu dire? Mais reconnaissonsle, poser comme on le fait généralement la question de la place de la philosophie, dans un cursus, dans une institution n'est-ce pas déplier d'emblée le strapontin du supplément d'âme? La présence en question me semble en effet, et d'expérience, une question d'acte? Et le pari qu'ouvre la création des IUFM, la philosophie ne pourra le tenir que de sa capacité à cet acte qu'est le dialogue, en l'occurrence la confrontation exigeante avec, entre autres, les sciences sociales et l'histoire. Pour assurer la possibilité même de cette philosophie en acte dans l'institution, il faudra de nouveau critiquer les prétentions abusives et les illusions de la science de l'éducation--de la croyance en la pédagogie comme science et comme technique appliquée.

Mais une telle entreprise critique n'a de sens que dans la reconnaissance de la complexité des relations entre le travail philosophique et les institutions dans lesquelles il s'effectue. C'est pourquoiavant de reprendre l'air connu de la polémique entre une philosophie assurée de sa clairvoyance et les sombres desseins des technologieset des sciences 
de l'éducation, je préférerais qu'on prenne d'abord le temps d'une polémique entre philosophes. Polémique que suscitent les prétentions abusives d'une philosphie un peu vite convaincue de ses vertus formatrices parce qu'assuré d' être le propriétaire exclusif de l'esprit critique. Car la philosophie est en situation et son discours ne se soutient que de reprendre patiemment et laborieusement les fils que déroulent une histoire et une sociologie de propre inscription dans le temps.

Sauf à faire confiance aux défaillances de la mémoire et à en conclure qu'on a toujours su et qu'on n'a jamais appris, force est de reconnaître que le temps, l'institution et la formation sont indissolublement liés, aussi loin qu'on remonte vers les conditions même de l'exercice de la pensée. Il s'agit bien ici d'enfance, de "l'institution des enfants", de l'enfance même du savoir. L'expérience singulière est depuis toujours confrontée à l'institution déjà-là. Tel est le drame de la culture dans lequel l'école devrait jouer le rôle d'instance régulatrice entre l'individuel et le social puisqu'elle est le lieu collectif de l'exercice du savoir, le seul lieu public où se puissent conjuguer la formation du sujet et l'institution du social.

Il faut comprendre à la fois que le drame individuel a lieu entre des rôles déjà inscrits dans l'ensemble institutionnel, que donc, depuis son début dans la vie, l'enfant procède, par la simple perception des soins qu'on lui donne et des ustensiles qui l'entourent, à un déchiffrement de signification, qui d'emblée généralise son drame propre en drame de sa culture et que, cependant, toute la conscience symbolique élabore en fin de compte ce que l'enfant vit ou ne vit pas, souffre ou ne souffre pas, sent ou ne sent pas, de sorte qu'il n'est pas un détail de son histoire la plus individuelle qui n'apporte quelque chose à cette signification sienne qu'il manifestera quand, ayant d'abord pensé et vécu selon qu'il croyait bon de le faire, et perçu d'après l'imaginaire de sa culture, il en vient enfin à renverser le rapport et à glisser dans les significations de sa parole et de sa conduite, à convertir en culture, jusqu'au plus secret de son expérience ${ }^{1}$

Je n'attends pas d'une philosophie intervenant dans l'institution et dans la formation qu'elle professe, qu'elle ajoute des leçons mais qu'elle fasse l'épreuve, au risque du présent, de la mise en perspective du métier d'instruire et d'instituer, dans son double rapport au temps du drame individuel et à la mémoire à long terme qu'est la culture. L'idée même d'école n'est rien d'autre que cette exigence que la culture soit à la fois une expérience singulière et un bien commun.

JEAN-CLAUDE POMPOUGNAC

${ }^{1}$ Maurice Merleau-Ponty. Signes, p. 123. 